\title{
CARCINOMA URETRAL CON EXTENSIÓN LOCORREGIONAL IMPORTANTE. ¿CÓMO MANEJAMOS UNA PATOLOGÍA INFRECUENTE?
}

\author{
A. SEGURA HUERTA, J. MOLINA SAERA, L. PALOMAR ABAD, L. PELLÍN ARIÑO, \\ A. GUERRERO ZOTANO, V. CALDERERO ARAGÓN
}

Servicio de Oncología Médica. Hospital La Fe. Valencia.

Actas Urol Esp. 28 (1): 57-61, 2004

\begin{abstract}
RESUMEN
CARCINOMA URETRAL CON EXTENSIÓN LOCORREGIONAL IMPORTANTE. ¿CÓMO MANEJAMOS UNA PATOLOGÍA INFRECUENTE?

El carcinoma primario de uretra es un tumor de escasa incidencia, ocupa menos del 0,1\% de las neoplasias genitourinarias. La mayoría de tumores corresponden a carcinomas escamosos, siendo mucho menos frecuentes los adenocarcinomas.

El tratamiento curativo es la cirugía y las técnicas dependen de la localización del tumor. La cirugía conservadora será de elección siempre que ésta no comprometa la curación. Determinadas localizaciones o extensión de enfermedad pueden requerir cirugía mutilante. En aquellos casos en los que no es posible un tratamiento quirúrgico se emplean otras estrategias terapéuticas (radioterapia o quimioterapia), sin que se disponga de estudios con número suficiente de pacientes que permitan establecer la mejor actitud terapéutica.

Presentamos el caso de una mujer de 35 años afecta de adenocarcinoma parauretral avanzado al diagnóstico. Ante la imposibilidad de recibir tratamiento quirúrgico fue tratada con quimioterapia más radioterapia. El tratamiento combinado produjo respuesta completa pero posteriormente apareció una recaída precoz que condicionó el exitus.
\end{abstract}

PALABRAS CLAVE: Adenocarcinoma uretral. Neoplasias uretrales. Tratamiento. Quimioterapia.

\section{ABSTRACT}

ADVANCED URETHRAL CARCINOMA. WHICH IS THE BEST MANAGEMENT OF A INFREQUENT DISEASE?

Urethral cancer is an uncommon tumor ( $<0.1 \%$ of all genitourinary neoplasms). Most of them are squamous carcinoma, adenocarcinomas are about $5 \%$ of all urethral cancer.

Surgery is the only curative treatment. Surgical technics depend of tumoral location and extension. Conservative surgery is elective if survival is not compromised. Extensive surgery is needed in large lessions. Chemotherapy (CT) and radiotherapy (RT) must be used in patients in wich surgery is not possible. Due to the low incidence of this neoplasm is not well established the best therapeutic approach. We present the case of a female (35 years old) with a diagnosis of urethral adenocarcinoma. The initial stage was IV due to non-regional lymph nodes metastases. Surgery was impossible and the patient received chemotherapy and radiotherapy. The patient achieved complete response with CT but a progression was observed in course of RT. The patient died due to sistemic progression.

KEY WORDS: Urethral adenocarcinoma. Urethral cancer. Treatment. Chemotherapy. 
$\mathrm{E}^{1}$ carcinoma primario de uretra es un tumor poco frecuente $(<0,1 \%$ de las neoplasias genitourinarias) $^{1,2}$. Aunque la uretra embriológicamente deriva de las mismas estructuras que el tracto genito-urinario y la vejiga, los tumores derivados de ellas son de diferentes histologías. La mayoría de tumores corresponden a carcinomas escamosos, siendo mucho menos frecuentes los adenocarcinomas ${ }^{3}$.

El tratamiento curativo es la cirugía, las técnicas dependen de la localización del tumor. Debe realizarse cirugía conservadora del órgano siempre que ésta no comprometa la curación ${ }^{3-5}$. En la enfermedad no localizada se emplean otras estrategias terapéuticas (radioterapia $(\mathrm{RT})^{6-9}$, quimioterapia $\left.(\mathrm{QT})^{10-14}\right)$, sin que se disponga de estudios con número suficiente de pacientes que permitan establecer la mejor actitud terapéutica.

Presentamos el caso de una mujer de 35 años afecta de adenocarcinoma parauretral avanzado al diagnóstico. Ante la imposibilidad de recibir tratamiento quirúrgico fue tratada con quimioterapia más radioterapia. El tratamiento combinado produjo una respuesta completa de la enfermedad pero posteriormente apareció una recaída sistémica precoz que condicionó el exitus.

\section{CASO CLÍNICO}

Mujer de 35 años sin antecedentes patológicos de interés que ingresó en mayo de 2001 en el Servicio de Medicina Interna por edematización en extremidades inferiores. A la exploración física destacaba edema con fóvea en ambos miembros inferiores y engrosamiento cutáneo sugestivo de linfedema crónico, se palpaban adenopatías inguinales bilaterales. El resto de la exploración física así como el hemograma y la bioquímica básica fueron normales. En una T.A.C. toraco-abdominopélvica se apreciaron adenopatías inguinales y retroperitoneales de gran tamaño (Fig. 1), no aparecieron lesiones en otros órganos.

Se realizó una PAAF de una adenopatía inguinal que fue informada como carcinoma. Tras realizar una exploración más detallada pareció objetivarse una lesión vulvar por lo que la paciente fue remitida al Servicio de Ginecología. Se realizó exploración ginecológica exhaustiva realizándose biopsias vulvares que fueron negativas. Se apreció infiltración tumoral en vagina que parecía

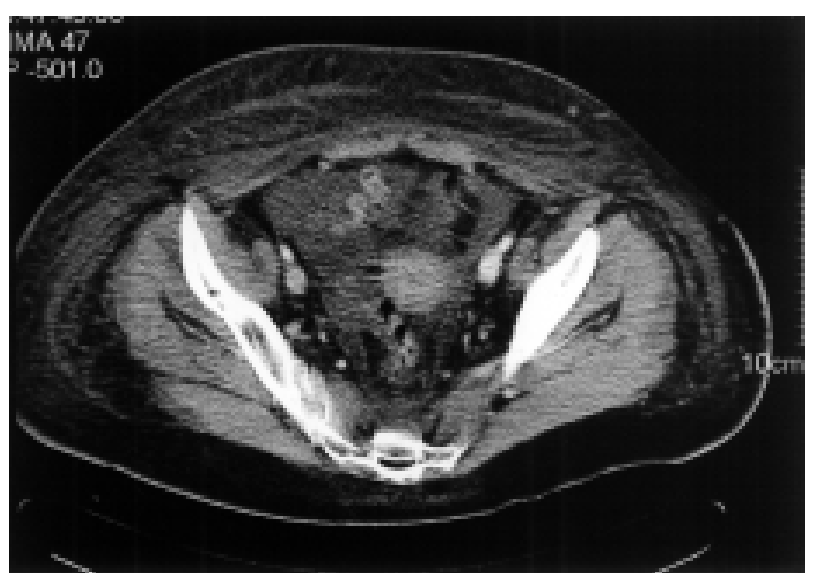

FIGURA 1. Masa parauretral y adenopatias pélvicas presentes al diagnóstico.

depender de la uretra. Una biopsia parauretral confirmó la existencia de un adenocarcinoma indiferenciado.

La enferma fue trasladada al Servicio de Urología donde se intentó realizar una cistoscopia sin que fuera posible debido a incapacidad de paso por uretra. Ante la extensión locorregional de la enfermedad (estadio IV por afectación ganglionar no regional), se descartó actitud quirúrgica y se consultó al Servicio de Oncología Médica el 27.07.01. Tras valoración clínica se consideró a la paciente candidata a tratamiento con QT. Se optó por el esquema PAC que comporta: cisplatino $70 \mathrm{mg} / \mathrm{m} 2$, adriamicina $50 \mathrm{mg} / \mathrm{m} 2$, ciclofosfamida $600 \mathrm{mg} / \mathrm{m} 2$, los tres citostáticos un solo día, ciclos cada tres semanas. Se plantearon 3-4 ciclos con posterior evaluación de respuesta $\mathrm{y}$ valoración de RT ganglionar.

Entre agosto y noviembre de 2001 recibió 4 ciclos de QT, objetivándose tras el tercero respuesta completa. Durante el tratamiento con QT requirió ingreso en tres ocasiones por toxicidad hematológica grado 4 con fiebre neutropénica que se resolvieron. Debido a la mala tolerancia hematológica se detuvo el tratamiento tras cuatro ciclos. Tras la evaluación de respuesta se consideró candidata a RT de consolidación. Inició dicho tratamiento en diciembre de 2001. Recibió un total de 55 Gy mediante fuentes de irradicación externa con un fraccionamiento convencional (1,8 Gy/día, 5 días a la semana).

En febrero de 2002 la paciente fue remitida desde RT ante la aparición de lesiones cutáneas que abarcaban ambos vacíos e hipogastrio. Se 
realizó una biopsia cutánea que confirmó la infiltración por adenocarcinoma. La paciente ingresó el 16.04.02 por deterioro del estado general con síndrome constitucional, disnea a mínimos esfuerzos y aumento de edemas. Se objetivó deterioro de las funciones hepática y renal junto a un derrame pleural bilateral (Fig. 2). Todo ello sugería progresión de enfermedad. Durante ese ingreso se produjo un deterioro clínico importante e irreversible que condicionó el exitus el 27.04.02.

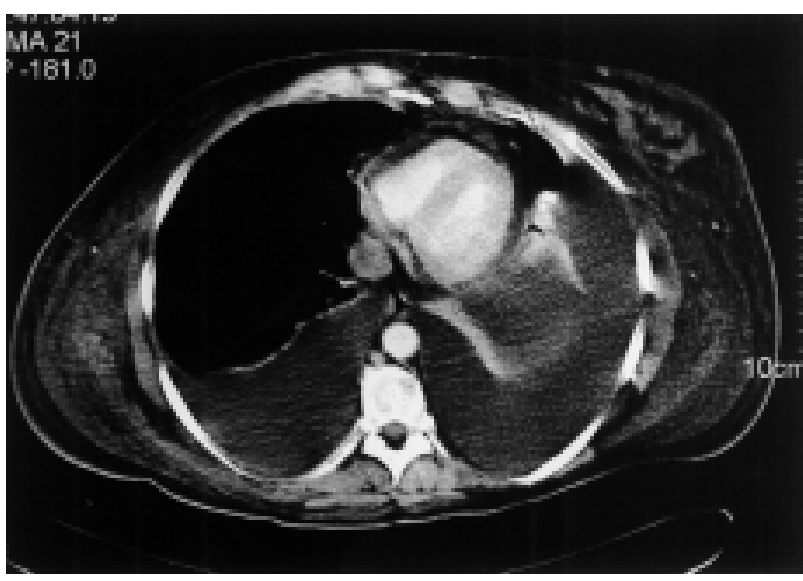

FIGURA 2. Derrame pleural secundario a metástasis. Situación clinica poco antes del fallecimiento de la paciente.

\section{DISCUSIÓN}

En la uretra pueden asentar tanto tumores benignos (adenomas nefrogénicos, papilomas, amiloidosis, hemangiomas, leiomiomas) como malignos. El carcinoma primario de uretra es un tumor poco frecuente. Su incidencia es mayor en mujeres que en hombres (4:1) y la edad de aparición es la sexta década de la vida. Es más frecuente en la raza caucásica ${ }^{8}$. Como posibles factores etiológicos se han relacionado la inflamación crónica y el virus del papiloma humano 16 y $18^{15}$.

Únicamente existen 1.200 casos de carcinoma de uretra descritos en mujeres y 600 en hombres. La mayoría de estos tumores son carcinomas de células escamosas y de células transicionales. Los adenocarcinomas son poco frecuentes: $18 \%$ de los carcinomas uretrales en mujeres y un 5\% en los hombres ${ }^{3}$, por ello ante el diagnóstico de adenocarcinoma de uretra siempre debe realizarse un estudio minucioso para descartar un posi- ble origen extrauretral. Sus características histológicas pueden ser idénticas a las del adenocarcinoma de colon y existe una variante de adenocarcinoma de células claras que se puede poner de manifiesto con anticuerpos frente a PSA y a la fosfatasa ácida prostática ${ }^{16,17}$.

Macroscópicamente puede aparecer como polipoide, indurado o como una úlcera superficial $^{18}$. La diseminación regional depende de la localización, los de uretra anterior drenan sobre todo en ganglios linfáticos inguinales y los de uretra posterior en linfáticos pélvicos ${ }^{19}$.

Clínicamente se presenta como uretrorragia, dispareunia en las mujeres o masa palpable uretral. En un $20 \%$ de pacientes la forma de presentación es como adenopatías inguinales palpables. No es frecuente la presentación como obstrucción urinaria $^{18}$.

El sistema de estadificación recomendado tanto por la American Joint Commission on Cancer como por la Unión Internacional Contra el Cáncer es el TNM ${ }^{20}$. El diagnóstico hoy en día se realiza fundamentalmente con cistouretroscopia y biopsia. En aquellos casos en los que el tumor sea productor de mucina debe diferenciarse ésta del gel empleado en la realización de la endoscopia. La ureterografía retrógada evidencia un defecto de replección en la uretra. La TAC y la RMN se emplean para la evaluación de la extensión local y a distancia ${ }^{18}$.

El tratamiento de estos tumores depende del sexo del paciente, de la extensión y de la localización tumoral.

En las mujeres la supervivencia a 5 años es del $75 \%$ en las lesiones de uretra anterior y únicamente del $10-17 \%$ en las de uretra posterior ${ }^{3}$. Las lesiones anteriores se tratan con cirugía radical (uretrectomía parcial o total), existe la posibilidad de realizar excisiones locales o con láser en tumores superficiales. El tratamiento con láser presenta menos complicaciones (hemorragia, cicatriz hipertrófica) pero se asocia a un mayor número de recaídas locales. Las lesiones de uretra posterior en mujeres requieren exenteración pélvica con derivación urinaria y disección ganglionar ilio-inguinal ${ }^{13}$.

En los varones la supervivencia a 5 años es del $22 \%$ en las lesiones de la uretra peneana y sólo del $10 \%$ en los tumores de uretra bulbo- 
membranosa. La penectomía parcial es la opción de tratamiento para los tumores distales. La excisión local seguida de radioterapia adyuvante es una opción que produce un elevado porcentaje de control de enfermedad en pacientes con tumores distales. Las lesiones de uretra bulbomembranosa se tratan con penectomía total, cistoprostatectomía, derivación urinaria y disección ganglionar ${ }^{5}$.

En tumores localizados inoperables la RT puede ser una alternativa curativa utilizando braquiterapia con Ir-192 en lesiones precoces y la RT externa en lesiones de mayor tamaño utilizando dosis de 45-50 Gy en 5 semanas $^{8}$. Si existe afectación de ganglios linfáticos se utilizan 60-65 Gy. En algunos pacientes con carcinoma de uretra clínicamente inoperable se ha utilizado la braquiterapia combinada con RT externa ${ }^{9}$. En mujeres con tumores del meato anterior, tratadas con RT combinada, se han conseguido supervivencias a 5 años superiores al 70\%, mientras que con carcinoma posterior sólo del 50\%. En cuanto a la RT en el varón, permite la preservación del órgano en las de uretra distal. Las lesiones precoces, como en la mujer, se pueden tratar con RT intracavitaria. Las lesiones más avanzadas son tratadas con una técnica que utiliza campos paralelos opuestos con el pene suspendido verticalmente mediante un catéter uretral. Esta técnica, sin embargo, no es útil para tratar adenopatías inguinales ${ }^{11,12}$.

La QT se reserva para la enfermedad avanzada habiendo sido utilizada únicamente en estudios no controlados debido a la baja incidencia de esta enfermedad. Se debe utilizar en lesiones con afectación de ganglios linfáticos pélvicos o inguinales, consiguiendo tasas de supervivencia a 5 años del 10-30\%. Los citostáticos más utilizados son el cisplatino, la bleomicina y el metotrexate, teniendo la monoterapia únicamente efecto paliativo. Con intención curativa se pueden emplear distintas combinaciones de estos agentes, siendo útil en ocasiones asociar RT de consolidación ${ }^{10-12}$.

En nuestro caso nos encontramos ante una mujer de 36 años afecta de un adenocarcinoma parauretral con extensión locorregional debido a afectación ganglionar inguinal y retroperitoneal (estadio IV por afectación ganglionar no regional), no fue posible un tratamiento local curativo. Dado que se trataba de una paciente joven nos planteamos tratamiento con quimioterapia más radioterapia. Con el esquema utilizado se alcanzó respuesta completa aunque se produjo progresión precoz de enfermedad mientras se encontraba en curso de radioterapia.

A pesar del resultado del tratamiento en nuestro caso, vemos como la QT puede tener un papel en este tipo de tumor. Los pacientes que no sean candidatos a tratamientos locales deben recibir quimioterapia y/o radioterapia si su estado general lo permite. La ausencia de protocolos estandarizados nos debe hacer pensar en la necesidad de incluir a los pacientes en estudios controlados que deberían ser multicéntricos y dada la escasa incidencia de esta enfermedad casi seguro internacionales.

\section{REFERENCIAS}

1. SRINIVAS V, KHAN SA.: Female urethral cancer. Int Urol Nephrol 1987; 19: 423-427.

2. SRINIVAS V, KHAN SA.: Male urethral cancer. A review. Int Urol Nephrol 1988; 20: 61-65.

3. HARTY JI.: Carcinoma of the female urethra. Decision Making in Oncology: evidence based management. New York, Churchill Livingstone 1977: 325-328.

4. BRACKEN RB, HENRY R, ORDÓÑEZ N.: Primary carcinoma of the male urethra. South Med J 1980; 73: 1003-1005.

5. HOPKINS SC, NAG SK, SOLOWAY MS.: Primary carcinoma of male urethra. Urology 1984; 23: 128133.

6. MICAILY B, DZEDA MF, MIYAMOTO CT, BRADY LW.: Brachytherapy for cancer of the female urethra. Semin Surg Oncol 1997; 13: 208-214.

7. KLEIN FA, ALI MM, KERSH R.: Carcinoma of the female urethra: combined iridium 192 interstitial and external beam radiotherapy. South Med $J$ 1987; 80: 1129-1132.

8. WEGHAUPT K, GERSTNER GJ, KUCERA H.: Radiation therapy for primary carcinoma of the female urethra: a survey over 25 years. Gynecol Oncol 1984; 17: 58-63.

9. KUETTEL MR, PARADA DS, HARTER KW, RODGERS JE, LYNCH JH.: Treatment of female urethral carcinoma in medically inoperable patients using external beam irradiation and high dose rate intracavitary brachytherapy. J Urol 1997; 157: 1669-1671.

10. EISENBERGER MA.: Chemotherapy for carcinomas of the penis and urethra. Urol Clin North Am 1992; 19: 333-338.

11. LUTZ ST, HUANG DT.: Combined chemoradiotherapy for locally advanced squamous cell carcinoma of the bulbomembranous urethra: a case report. $J$ Urol 1995; 153: 1616-1618.

12. TRAN LN, KRIEG RM, SZABO RJ.: Combination chemotherapy and radiotherapy for a locally advanced squamous cell carcinoma of the urethra: a case report. J Urol 1995; 153: 422-423. 
13. NARAYAN P, KONETY B.: Surgical treatment of female urethral carcinoma. Urol Clin North Am 1992; 19: 373-382.

14. BEDUSCHI MC, WISHNOW KY, OESTERLING JE.: Urethral carcinoma: diagnosis and staging. In Oesterling JE, Richie JP (eds) Urologic Oncology: Philadelphia, Pennsylvania: WB Saunders 1997: 561-571.

15. WIENER JS, WALTHER PJ.: The association of oncogenic human papilomavirus with urologic malignancy. The controversies and clinical implicatons. Surg Oncol Clin North Am 1995; 4: 257-276.

16. STAGIER J, VAN POPPEL H, MERTENS V, GEOBES K, BAERT L.: Adenocarcinoma of the rectum with a solitary metastasis to the urethra in a female. Eur J Surg Oncol 1994; 20: 696-697.

17. ASSIMOS DG, O'CONOR VJ Jr.: Clear cell adenocarcinoma of the urethra. J Urol 1984; 131: 540541.
18. MOSTOFI FK, DAVIS CJ Jr, SESTERHENN IA.: Carcinoma of the male and female urethra. Urol Clin North Am 1992; 19: 347-358.

19. CARROLL PR, DIXON CM.: Surgical anatomy of the male and female urethra. Urol Clin North Am 1992; 19: 339-346.

20. FLEMING ID, COOPER JS, HENSON DE, HUTTER RVP et al.: Urethra. In AJCC Cancer Staging Manual, $5^{\text {th }}$ edition. Philadelphia, Pennsylvania: Lippincott-Raven 1997.

Dr. A. Segura Huerta

Servicio de Oncología. Hospital Univ. La Fe

Avda. de Campanar, 21

46009 Valencia

(Trabajo recibido el 2 septiembre 2003) 